\title{
A Fault Detection Approach Using Variational Mode Decomposition, L-kurtosis and Random Decrement Technique for Rotating Machinery
}

\author{
Hui Liu, Zhiyu Shi* \\ State Key Laboratory of Mechanics and Control of Mechanical Structures, Nanjing University of Aeronautics and Astronautics, Nanjing, \\ China
}

Email address:

zyshi@nuaa.edu.cn (Zhiyu Shi)

${ }^{*}$ Corresponding author

\section{To cite this article:}

Hui Liu, Zhiyu Shi. A Fault Detection Approach Using Variational Mode Decomposition, L-kurtosis and Random Decrement Technique for Rotating Machinery. International Journal of Mechanical Engineering and Applications. Vol. 8, No. 1, 2020, pp. 16-26.

doi: 10.11648/j.ijmea.20200801.13

Received: January 6, 2020; Accepted: January 21, 2020; Published: January 31, 2020

\begin{abstract}
Fault detection of rotating machinery under heavy noise background, is a significant but difficult issue, and traditional fault detection approaches are difficult to apply. To address this problem, a novel approach that combines variational mode decomposition (VMD), L-Kurtosis and random decrement technique (RDT) is proposed, which procedures are summarized as follows. First, the raw vibration signal collected from the rotating component is decomposed using VMD into a set of intrinsic mode functions (IMFs), and the noise components can be separated from the raw signal. Second, the L-Kurtosis indicator is introduced to solve the problem that the fault information is difficult to track, and the optimal intrinsic mode function (IMF) can be determined according to the maximum L-Kurtosis value. Then, RDT is further employed to purify the optimal IMF to eliminate the other unknown interference sources. Finally, a Hilbert envelope spectrum analysis is used for detecting the fault type. In order to validate the proposed approach, the numerical simulations and real experimental investigations about rolling element bearing and gear are conducted. The results illustrate that the proposed approach can effectively detect faults of rotating components.
\end{abstract}

Keywords: Rotating Machinery, Fault Detection, Variational Mode Decomposition, L-Kurtosis, Random Decrement Technique

\section{Introduction}

Rolling element bearing and gear are the key components used in modern rotating machinery and play the increasingly important role. Once the breakdowns of these key rotating components occur and with the development of fault, may lead to high economic loss, huge security problems, even heavy casualties. Due to the importance, the detection of the faults in rotating components has attracted wide attention, and it is of particular importance to expand related research to guarantee the safety of machine operations and staffs [1-6].

Proven as the most effective technique for condition monitoring and fault diagnosis through lots of experimental researches, vibration analysis $[7,8]$ has developed vigorously. The vibration signals collected from faulty bearing and gear carry enormous faulty information, which is manifested as a series of periodic impact impulses, but usually contaminated by noise, and the extracted feature frequency can not characterize the faulty type. Nowadays, plenty of signal processing techniques has been proposed, and mainly divided into two categories [9], i.e., the intelligent classification and faulty feature frequency extraction. For the first category, it relies on intelligent technique to distinguish whether the state of the rotating component is healthy or faulty, such as deep belief network (DBN) [10], hidden Markov model (HMM) [11] and convolutional neural network (CNN) [12], etc. However, the number of samples for different fault types is far below the demand, which has largely limited the development of such techniques. The main core of the another category is to eliminate the noise and other unknown interference sources, 
and extract the faulty feature frequency. The Hilbert envelope analysis $[13,14]$ is one of the beneficial tools for identifying vibration impact, given such benefit, it has been applied in detecting the fault type for mechanical system. However, its performance depends greatly on the selection of the denoising technique.

Among the available denoising techniques, variational mode decomposition (VMD) [15] has been developed rapidly due to its potential in detecting faults. VMD is an adaptive technique that can decompose the non-stationary vibration signal into a series of independent intrinsic mode functions (IMFs) that represent the natural oscillatory modes of the raw signal. Recently, many researchers has investigated its performance. Zheng et al. [16] optimized VMD technique and applied it to detect faults in rotating machinery, and the results shows that the optimized technique can extract the knocking abnormal noise component effectively. Gu et al. [17] proposed a hybird approach combining VMD and Teager energy operator, and successfully applied to the early fault detection of the rolling element bearing. Du et al. [18] proposed a novel technique named fractional iterative variational mode decomposition based on VMD, and the result verify its obvious advantage in processing the noisy signals and even signals containing weak components. Yang et al. [19] combined VMD and phase space parallel factor analysis technique, and the result indicate that the combination has good performance in detecting weak fault signal of the rotating components.

However, the selection of the intrinsic mode function (IMF) usually depends on human experience, and a inappropriate selection strongly influences the implementation 1 of the subsequent steps. In order to accurately analyze an IMF containing the faulty information and avoid the problem of selection, the L-Kurtosis indicator is introduced. L-Kurtosis is a fourth-order L-moment, which has obvious advantage in parameter estimation and robustness to outliers compared with kurtosis. Withers CS and Nadarajah S [20] made a comparison of several indicators and proved that the superiority of the L-Kurtosis indicator. Liu et al. applied L-Kurtosis into the fault detection of rolling element bearing and achieve good results [21].

For the optimal IMF, it mainly consists of two parts, i.e., the random part and deterministic part. The random part represents the noise and other unknown interference sources, and the deterministic part represents the periodic impact impulse signal containing faulty information. As a time-domain technique, Random decrement technique (RDT) is usually used to extract the structure modal properties from the vibration signal [22-27]. Huang et al. [28] introduced RDT to identify the Vortex-induced vibration response automatically from the massive acceleration response and achieved good result. Here, RDT is used to remove the random part from the optimal IMF through averaging enough sample responses, and the purified signal (periodic impulse signal) containing the faulty information can be extracted [29-30]. In view of the limitations of the traditional fault detection approaches, combining VMD, L-Kurtosis and RDT might be suitable to detect the faults of rotating components.

In this paper, VMD is employed to decompose the vibration signal into a set of IMFs, L-Kurtosis is introduced to select the optimal IMF, RDT is applied to further extract the purified signal containing the faulty information, which is demodulated using a Hilbert envelope analysis to extract the faulty feature frequency. Therefore, the remainder pare of this paper is compiled as follows. Section II introduces the proposed approach and reviews the bases of VMD, L-Kurtosis and RDT in details, respectively. Section III indicates the proposed approach by simulated data. In Section IV, the real experimental data collected from the faulty bearings and gears are further analyzed to verify the proposed approach. The whole paper has been concluded in Section V.

\section{The Proposed Approach}

\subsection{Variational Mode Decomposition}

In terms of signal decomposition, VMD [15] has the obvious advantage, which can decompose the complex and multi-component signal into a set of IMFs. Based on the signal analysis theory, the vibration signal $y(t)$ can be represented by a series of IMFs $u_{k}$ preserving the natural oscillatory modes of the raw signal. The signal $y(t)$ can be expressed as follows [15]:

$$
y(t)=\sum_{k} u_{k}
$$

The most critical aspect of VMD is the problem of solving the constrained variational model [31-32], which is given by:

$$
\min _{\left\{u_{k}\right\}\left\{\omega_{k}\right\}}\left\{\sum_{k}\left\|\partial_{t}\left[\left(\delta(t)+\frac{j}{\pi t}\right) * u_{k}(t)\right] e^{-j \omega_{k} t}\right\|_{2}^{2}\right\}
$$

where $\left\{u_{k}\right\}$ and $\left\{\omega_{k}\right\}$ contain all IMFs and corresponding center frequencies. $\|-\|_{2}, \delta$ and * represent the Euclid norm, Dirac distribution and convolution operator, respectively.

The key to solve the problem is that how to convert the constrained problem into an unconstrained problem, therefore a quadratic penalty term $\alpha$ and Lagrangian multipliers $\lambda(t)$ are introduced. The model (show in (2)) is updated to the following form:

$$
\begin{aligned}
& L\left(\left\{u_{k}\right\},\left\{\omega_{k}\right\}, \lambda\right)= \\
& \alpha \sum_{k}\left\|\partial_{t}\left[\left(\delta(t)+\frac{j}{\pi t}\right) * u_{k}(t)\right] e^{-j \omega_{k} t}\right\|_{2}^{2} \\
& +\left\|y(t)-\sum_{k} u_{k}(t)\right\|_{2}^{2}+\left\langle\lambda(t), y(t)-\sum_{k} u_{k}(t)\right\rangle
\end{aligned}
$$

Meanwhile, the equations of $u_{k}, \omega_{k}$ and $\lambda(t)$ are updated as follows: 


$$
\begin{gathered}
\hat{u}_{k}^{n+1}(\omega)=\frac{\hat{y}(\omega)-\sum_{i>k} \hat{u}_{i}(\omega)+\frac{\hat{\lambda}(\omega)}{2}}{1+2 \alpha\left(\omega-\omega_{k}\right)^{2}} \\
\hat{\omega}_{k}^{n+1}=\frac{\left.\int_{0}^{\infty} \omega \hat{u}_{k}(\omega)\right|^{2} \mathrm{~d} \omega}{\int_{0}^{\infty}\left|\hat{u}_{k}(\omega)\right|^{2} \mathrm{~d} \omega} \\
\hat{\lambda}^{n+1}(\omega)=\hat{\lambda}^{n}(\omega)+\tau\left(\hat{y}(\omega)-\sum_{k} \hat{u}_{k}^{n+1}(\omega)\right)
\end{gathered}
$$

where $\tau$ represents the update parameter and the mark $\wedge$ represents the updated value.

\subsection{L-Kurtosis}

L-Kurtosis is more accurate in parameter estimation and more robust to outliers compared with kurtosis. Here, L-Kurtosis is introduced [21] to track the faulty information.

$\left\{u_{q}\right\}=\left\{u_{1}, \ldots, u_{q}\right\}$ represents the continuous independent sample with the cumulative distribution $F(u)$, $\left\{u_{q: q}\right\}=\left\{u_{1: q}, \ldots u_{q: q}\right\}$ represents corresponding order statistics. The $r$ th L-mocment $\eta_{r}$ is given by:

$$
\eta_{r}=\frac{1}{r} \sum_{k=0}^{r-1}(-1)^{r}\left(\begin{array}{c}
r-1 \\
k
\end{array}\right) E\left(u_{r-k: r}\right), r=1,2, \ldots
$$

$E\left(u_{r-k: r}\right)$ can be described as follows:

$$
\begin{aligned}
& E\left(u_{r-k: r}\right)= \\
& \frac{r !}{(r-k-1) ! k !} \int_{0}^{1} u\left[F(u)^{r-\mathrm{k}-1}\right][1-F(u)]^{k} d F(u)
\end{aligned}
$$

And the first four order L-moment can be obtained:

$$
\begin{gathered}
\eta_{1}=E U=b_{0}=\int_{0}^{1} u d F(u) \\
\eta_{2}=\frac{1}{2} E\left(u_{2: 2}-u_{1: 2}\right)=2 b_{1}-b_{0} \\
=\int_{0}^{1} u(2 F(u)-1) d F(u) \\
\eta_{3}=\frac{1}{3} E\left(u_{3: 3}-2 u_{2: 3}+u_{1: 3}\right) \\
=6 b_{2}-6 b_{1}+b_{0} \\
=\int_{0}^{1} u\left(6 F^{2}(u)-6 F(u)+1\right) d F(u)
\end{gathered}
$$

$$
\begin{aligned}
& \eta_{4}=\frac{1}{4} E\left(u_{4: 4}-3 u_{3: 4}+3 u_{2: 4}-u_{1: 4}\right) \\
& =20 b_{3}-30 b_{2}+12 b_{1}-b_{0} \\
& =\int_{0}^{1} u\left(20 F^{3}(u)-30 F^{2}(u)+12 F(u)-1\right) d F(u)
\end{aligned}
$$

in which $b_{i}=\int_{0}^{1} u F(u)^{i} d F(u), i=0,1,2,3$ is the $i$ th order weighted moment.

The calculation equation of the L-Kurtosis value $L K$ is presented as follows:

$$
L K=\frac{E\left(u_{4: 4}\right)-E\left(u_{1: 4}\right)-3\left[E\left(u_{3: 4}\right)-E\left(u_{2: 4}\right)\right]}{2\left[E\left(u_{2: 2}\right)-E\left(u_{1: 2}\right)\right]}
$$

\subsection{Random Decrement Technique}

In order to further eliminate other unknown interference sources, RDT is employed to the optimal IMF [29, 30]. Here, a suitable threshold level is applied to divide equally the optimal IMF into a series of segments $H$ with the same initial condition $\left(u_{i}\left(t_{i}\right)=u_{s}=\right.$ const. $\left., i=1,2, \ldots, H\right)$, and the length of division is $\mu$. By averaging all the segments $H$, the random decrement can be extracted and it can be expressed by:

$$
u(\mu)=\frac{1}{H} \sum_{i=1}^{H} u_{i}\left(t_{i}+\mu\right)
$$

Figure 1 shows the flowchart of the proposed approach, which mainly consists of the following three steps:

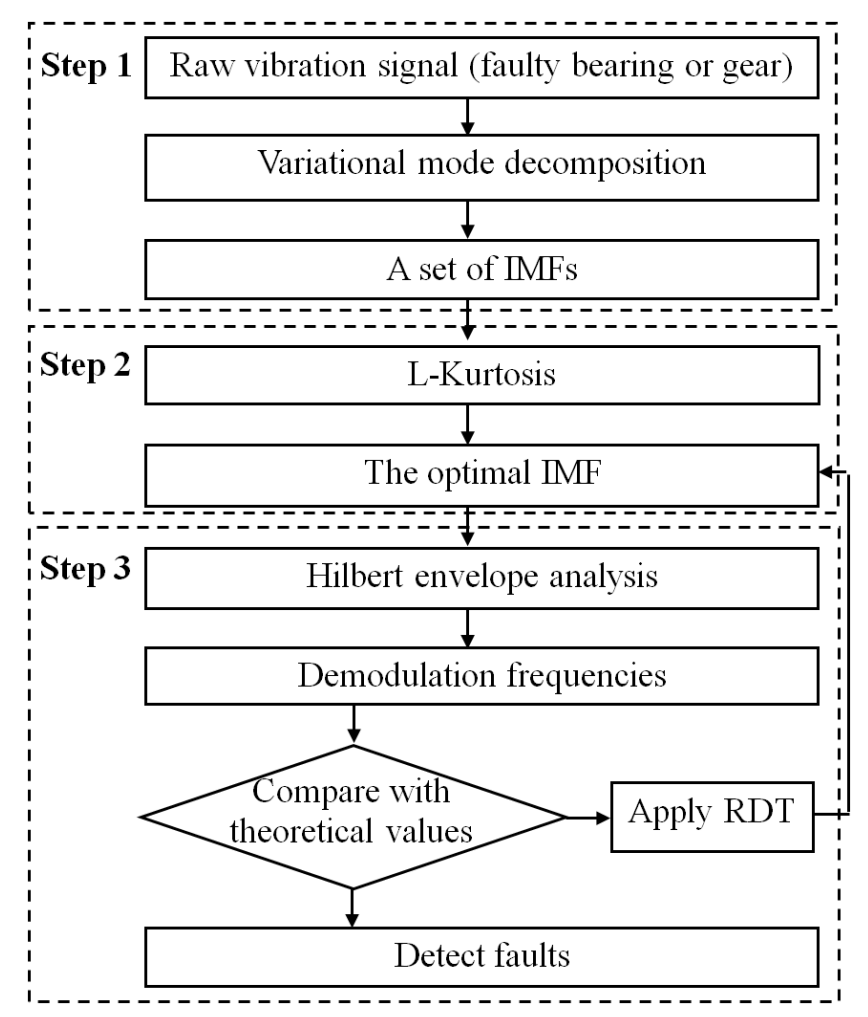

Figure 1. The flowchart of the proposed approach. 
(1) Decompose the raw signal using VMD.

For the simulated and real experimental signal, the VMD technique is employed and the signal is decomposed into a set of IMFs. In this step, the effect of the noise can be almost removed.

(2) Track the faulty information using L-Kurtosis.

An unsuitable IMF strongly influences the detection results and the selection of the optimal IMF is not easily possible. Aiming at this problem, the L-Kurtosis indicator is introduced in this work and the optimal IMF can be determined through the maximum L-Kurtosis value.

(3) Purify the optimal IMF using RDT and obtain the detect result.

For the optimal IMF, a Hilbert envelope spectrum analysis is used to demodulate the faulty feature frequency. If the demodulated frequency matches theoretical faulty feature frequency, the fault type of the rotating components (rolling element bearing and gear) can be determined. If not, RDT is further applied to purify the optimal IMF, the other unknown interference sources can be eliminated, and the detect result can be obtained by repeating Hilbert envelope spectrum analysis.

\section{Numerical Simulation}

To verify the capability of the proposed approach in analyzing the low signal-to-noise ratio signal, a vibration signal $y(t)$ corresponding to the rotating component is simulated in this section and its mathematical model is given as follows:

$$
y(t)=x(t+T)+z(t)+n(t)
$$

where $x(t)$ and $T$ represent the impulse response signal and impulse period, $z(t)$ represents the interference signal related to the rotating frequency, $n(t)$ represents the noise interference component.

The impulse response signal $x(t)$ is expressed by:

$$
x(t)=e^{-S t} \cos \left(2 \pi f_{n} t\right)
$$

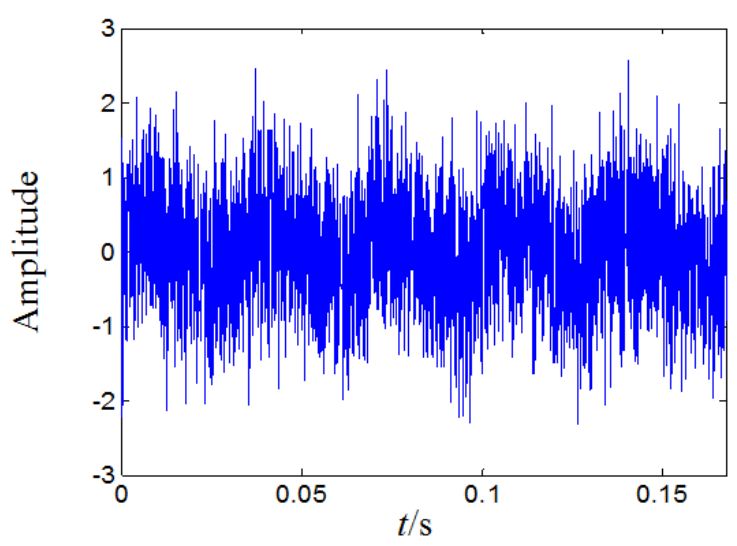

(a) where $S$ and $f_{n}$ represent the attenuation coefficient and natural frequency of rotating component, respectively. The attenuation coefficient $S$ is defined as follow:

$$
S=2 \pi f_{n} \gamma
$$

in which $\gamma$ represents the damping ratio.

The signal $z(t)$ is defined as

$$
z(t)=5 \sin \left(2 \pi f_{0} t\right)+1.5 \sin \left(4 \pi f_{0} t\right)+0.5 \sin \left(6 \pi f_{0} t\right)
$$

where $f_{0}$ represent the shaft rotating frequency, respectively.

The relevant parameters of the simulated signal are supposed as follows: $T=0.01 \mathrm{~s}, \quad S=500, f_{n}=4000 \mathrm{~Hz}$, $\gamma=0.019894, P=0.01, f_{0}=30 \mathrm{~Hz}$, and the corresponding faulty feature frequency is $100 \mathrm{~Hz}$. The noise component $n(t)$ is a standard normal distribution with standard deviation 3 , the sampling frequency $f_{s}$ is set to $20480 \mathrm{~Hz}$ and the sampling points $N$ is 13824 .

The time domain waveform of the simulated signal and its Hilbert envelope spectrum is presented in Figure 2. From Figure 2, it can be seen that the periodic impulses of the fault are mixed with the heavy noise, and the faulty feature frequency is submerged. In order to eliminate the noise interference, VMD is employed and the decomposition result of the simulated signal is shows in Figure 3. As shown in Figure 3, none of the decomposed IMFs exhibit the periodic impact characteristic clearly. The selection of the IMF containing the faulty information depends on manual experience in a general way and an unsuitable IMF strongly influences the implementation 1 of the subsequent steps. Aiming at this problem, the L-Kurtosis indicator is introduced and the relevant L-Kurtosis values is shown in Table 1 .

From Table 1, the L-Kurtosis values for the decomposed IMFs are 2.2334, 3.0476, 3.0989, 2.8546 and 3.0301, respectively. The maximum L-Kurtosis value corresponds to IMF3 and its Hilbert envelope spectrum is shown in Figure 4 (a). As shown in Figure 4 (a), the faulty feature frequency $(100 \mathrm{~Hz})$ and its harmonics are submerged by other unknown frequency components, such as $91.86 \mathrm{~Hz}$ and $171.7 \mathrm{~Hz}$, etc. Therefore, RDT is performed to IMF3 for further purifying.

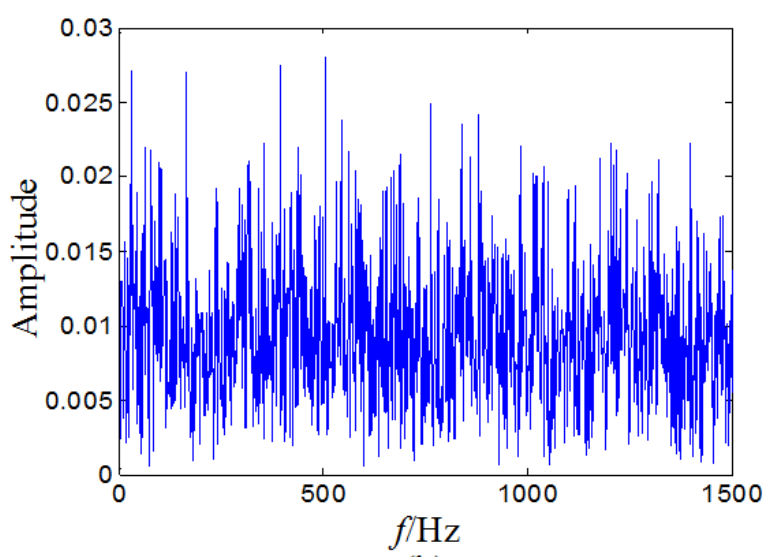

(b)

Figure 2. The time domain waveform of the simulated signal and corresponding Hilbert envelope spectrum. 

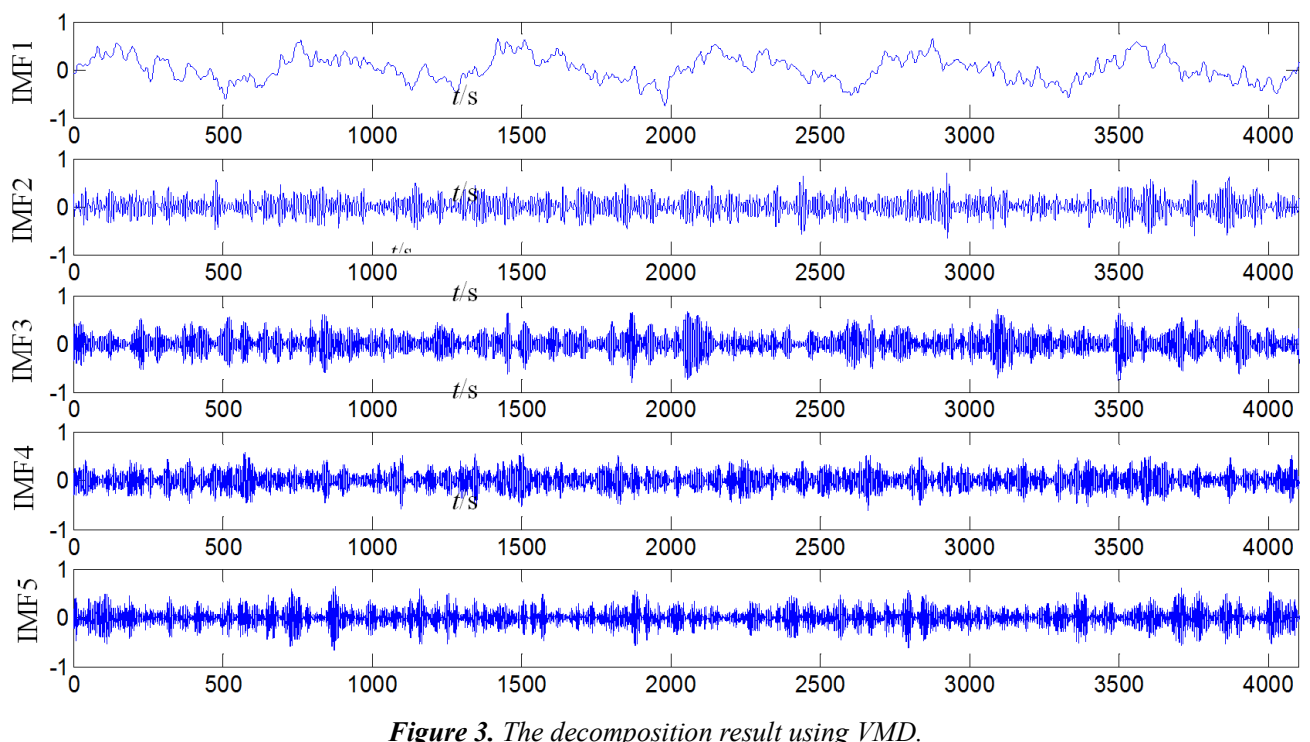

Figure 3. The decomposition result using $V M D$.

Table 1. The corresponding L-Kurtosis values.

\begin{tabular}{|c|c|c|c|c|c|}
\hline IMF & IMF1 & IMF2 & IMF3 & IMF4 & IMF5 \\
\hline L-Kurtosis value & 2.2334 & 3.0476 & 3.0989 & 2.8546 & 3.0301 \\
\hline
\end{tabular}

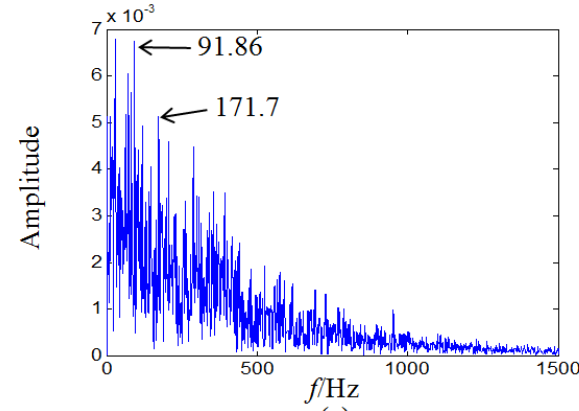

(a)

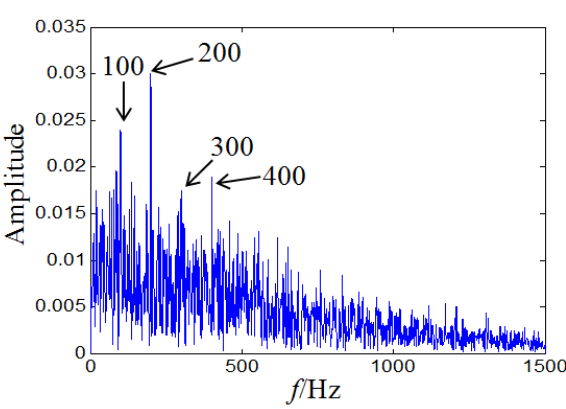

(b)

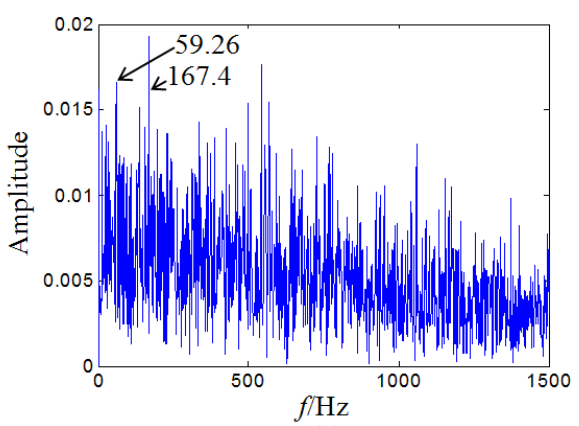

(c)

Figure 4. The Hilbert envelope spectrum of the (a) the optimal IMF, (b) the purified signal, (c) the combination of IITD, L-kurtosis and RDT.

The Hilbert envelope spectrum of the purified signal is shown in Figure 4 (b). In addition, in order to further verify the validity of the proposed approach, improved intrinsic time-scale decomposition (IITD) technique is introduced and the result of the combination of IITD, L-kurtosis and RDT is shown in Figure 4 (c). Comparing Figure 4 (a), Figure 4 (b) and Figure 4 (c), it can be seen that the faulty feature frequency $(100 \mathrm{~Hz})$ and its $2-4$ harmonics $(200 \mathrm{~Hz}, 300 \mathrm{~Hz}, 400 \mathrm{~Hz})$ are more clearly extracted in Figure 4 (b), the fault is detected successfully. Through the above analysis, the performance and the necessity of the combination of VMD, L-Kurtosis and RDT is indicated.

\section{Experimental Investigations}

In this section, real experimental investigations on the rolling element bearings and gear are conducted to further evaluate the ability of the proposed approach. The schematic of the machinery fault simulator test rig [6] is shown in Figure 5, which mainly includes the speed monitor, manual speed governor, speed sensors, motors, spindles, a computer with VQ data acquisition software and acceleration sensors. In order to simulate the inner race fault, outer race fault and the broken teeth fault, the single point defects are produced by electro-discharge machining on the corresponding positions in the bearings and gear, which are shown in Figures 6 (a), (b) and (c), respectively. The sampling frequency $f_{s}$ is set to $25.6 \mathrm{kHz}$ and the vibration signal points collected is 8192 .

The rotating components used in the experiment are the rolling element bearings of type ER-12K and bevel gear, respectively. The geometric parameters of the rolling element bearing are listed in Table 2 .

The shaft rotating frequency $f_{\text {shaft }}$ in the inner race fault experiment is set to $29.87 \mathrm{~Hz}$ and the ball pass frequency of inner race (BPFI) [33] is calculated using the following equation:

$$
B P F I=\frac{N_{b}}{2} f_{\text {shaft }}\left(1+\frac{B_{d}}{P_{d}} \cos \alpha\right)=147.8 H z
$$

The shaft rotating frequency $f_{\text {shaft }}$ in the outer race fault 
experiment is set to $29.87 \mathrm{~Hz}$ and the ball pass frequency of the outer race (BPFO) [33] is calculated using the following equation:

$$
B P F O=\frac{N_{b}}{2} f_{\text {shaft }}\left(1-\frac{B_{d}}{P_{d}}\right) \cos \alpha=91.15 H z
$$

The shaft rotating frequency $f_{\text {shaft }}$ in the broken teeth experiment is set to $29.40 \mathrm{~Hz}$ and the gear broken teeth fault manifests the shaft rotating frequency $f_{\text {shaft }}$ and its harmonics [34]. The number of gear teeth $z$ is 18 , and the mesh frequency $f_{m}$ is calculated using the following equation:

$$
f_{m}=z \times f_{\text {shaft }}=18 \times 29.40=529.2 \mathrm{~Hz}
$$

\subsection{Bearing Inner Race Fault Detection}

The time domain waveform of the raw vibration signal and corresponding Hilbert envelope spectrum are presented in Figure 7. As shown in Figure 7, the periodic impulse signal and the faulty feature frequency which can manifest the faulty type are submerged due to the heavy noise and other unknown interference sources, only the shaft rotating frequency and its 2-3 harmonics $(29.63 \mathrm{~Hz}, 59.26 \mathrm{~Hz}, 88.89 \mathrm{~Hz})$ appear. Hence, the proposed approach is performed.

VMD is employed to remove the noise component and the decomposition result of the raw vibration signal is shown in Figure 8. From Figure 8, it can be seen that the arrangement of the decomposed IMFs is disorderly and it is difficult to determine the optimal IMF containing the faulty information.

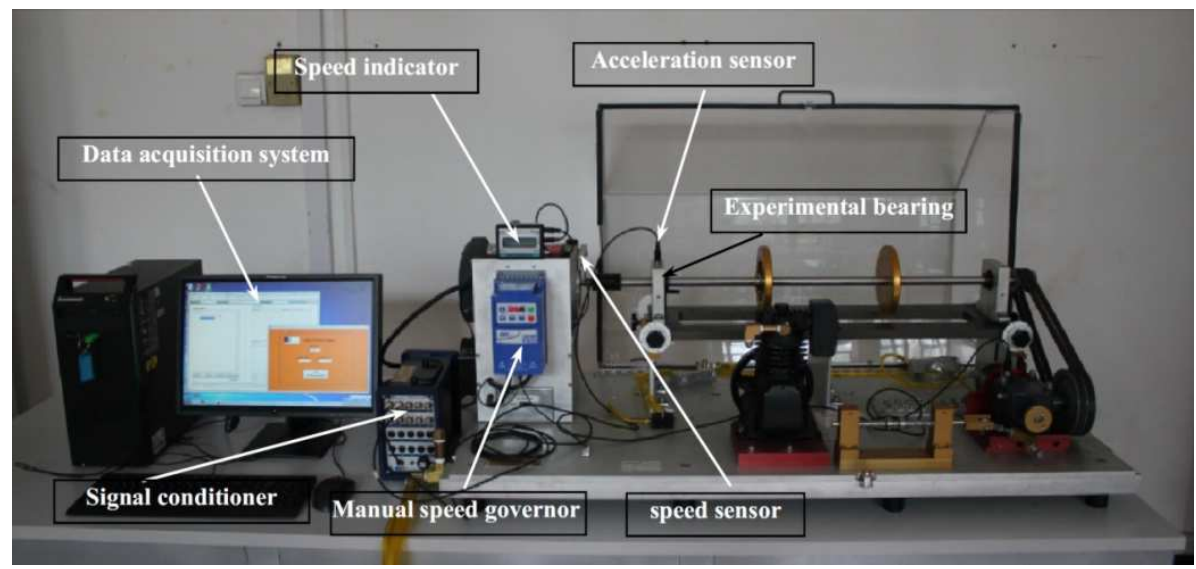

Figure 5. The schematic of the test rig.

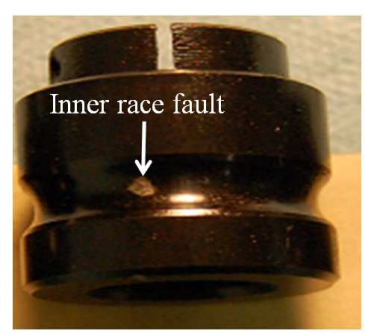

(a)

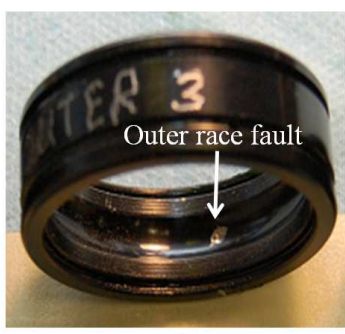

(b)

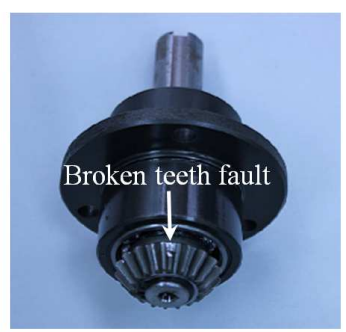

(c)

Figure 6. The bearing with (a) inner race fault, (b) outer race fault, (c) the gear with broken teeth fault.

Table 2. Geometric parameters of ER-12K bearing.

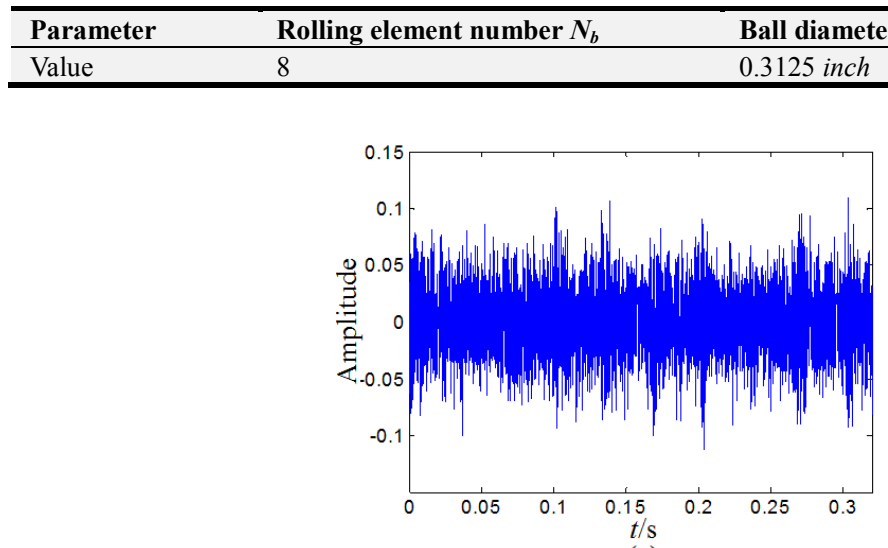

(a)

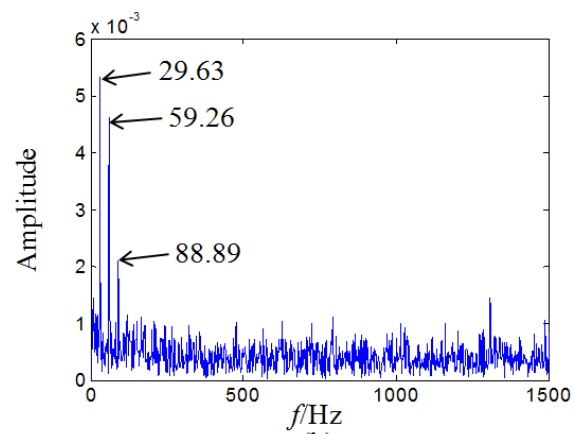

(b)

Figure 7. The time domain waveform of the raw vibration signal and corresponding Hilbert envelope spectrum. 
Aiming at this problem, the L-Kurtosis indicator is introduced and used for each decomposed IMF, and the corresponding L-Kurtosis value is shown in Table 3. From Table 3, it can be seen that the optimal IMF is IMF3 and the corresponding Hilbert envelope spectrum is shown in Figure 9 (a). From Figure 9 (a), the shaft rotating frequency $(29.63 \mathrm{~Hz})$ and its 2-4 harmonics $(63.59 \mathrm{~Hz}, 88.89 \mathrm{~Hz}, 118.5 \mathrm{~Hz})$ is appear, but the faulty feature frequency is heavily submerged and can not be extracted effectively. For this, RDT is further employed to eliminate the other interference sources from the optimal
IMF and Figure 9 (b) shows the Hilbert envelope spectrum of the purified signal. And the result of the combination of IITD, L-kurtosis, RDT is shown in Figure 9 (c). Comparing Figure 9 (a), Figure 9 (b) and Figure 9 (c), the faulty feature frequency $(148.7 \mathrm{~Hz}$ ) are extracted effectively in Figure 9 (b). Meanwhile, the extracted frequency $(148.7 \mathrm{~Hz})$ is matched with the theoretical calculation value (show in (19)). Based on the above analysis, the inner race fault of the bearing is successfully detected and the performance of the proposed approach is indicated.
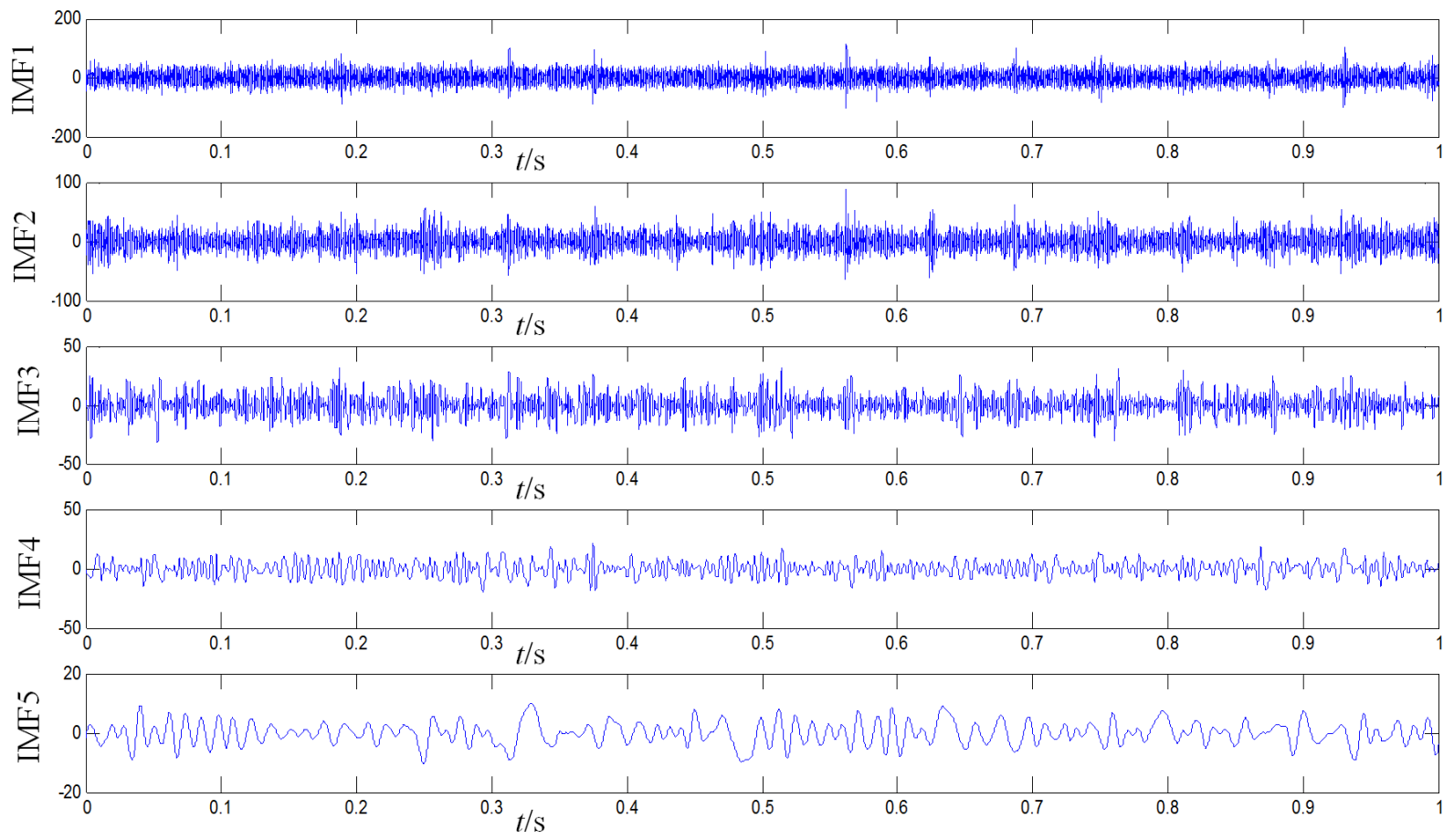

Figure 8. The decomposition result using VMD.

Table 3. The corresponding L-Kurtosis values.

\begin{tabular}{llllll}
\hline IMF & IMF1 & IMF2 & IMF3 & IMF4 & IMF5 \\
\hline L-Kurtosis value & 3.0615 & 5.2203 & 3.0928 & 3.4851 & 3.8042 \\
\hline
\end{tabular}

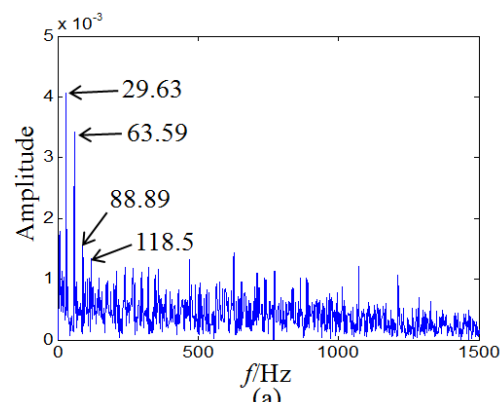

(a)

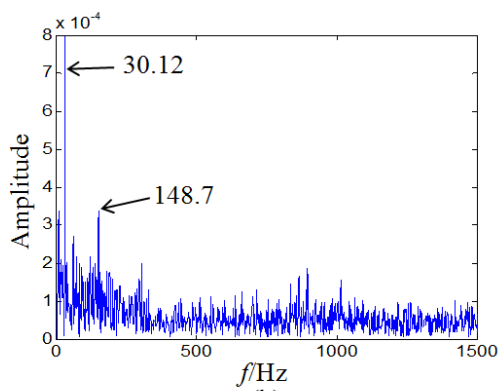

(b)

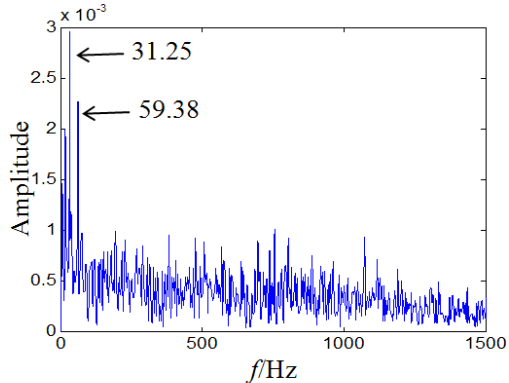

(c)

Figure 9. The Hilbert envelope spectrum of (a) the optimal IMF, (b) the purified signal, (c) the combination of IITD, L-kurtosis and RDT.

\subsection{Bearing Outer Race Fault Detection}

The time domain waveform of the raw vibration signal and corresponding Hilbert envelope spectrum are presented in Figure 10. From Figure 10 (b), the faulty feature frequency $(87.04 \mathrm{~Hz})$ is extracted but it is submerged by numerous frequency components, such as $29.63 \mathrm{~Hz}, 64.81 \mathrm{~Hz}$ and $116.7 \mathrm{~Hz}$, etc. Therefore, the proposed approach is applied.

Figure 11 shows the decomposition result using VMD and the relative L-Kurtosis values of the decomposed IMFs are shown in Table 4. From Table 4, the optimal IMF is IMF4 according to the maximum L-Kurtosis value and its Hilbert 
envelope spectrum is shown in Figure 12 (b).

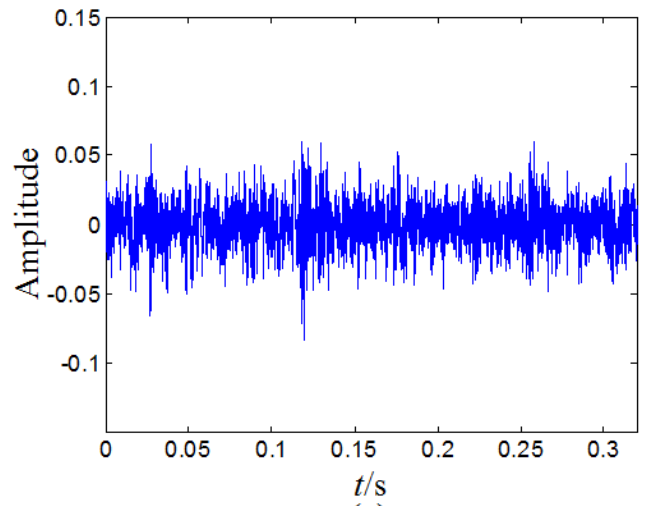

(a)

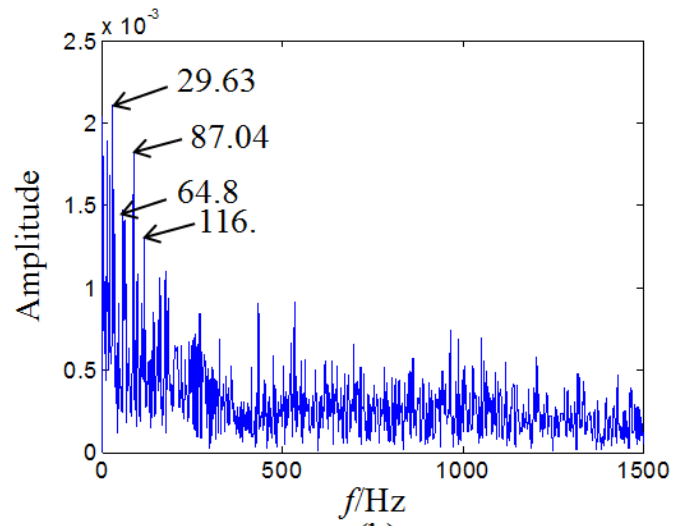

(b)

Figure 10. The time domain waveform of the raw vibration signal and corresponding Hilbert envelope spectrum.
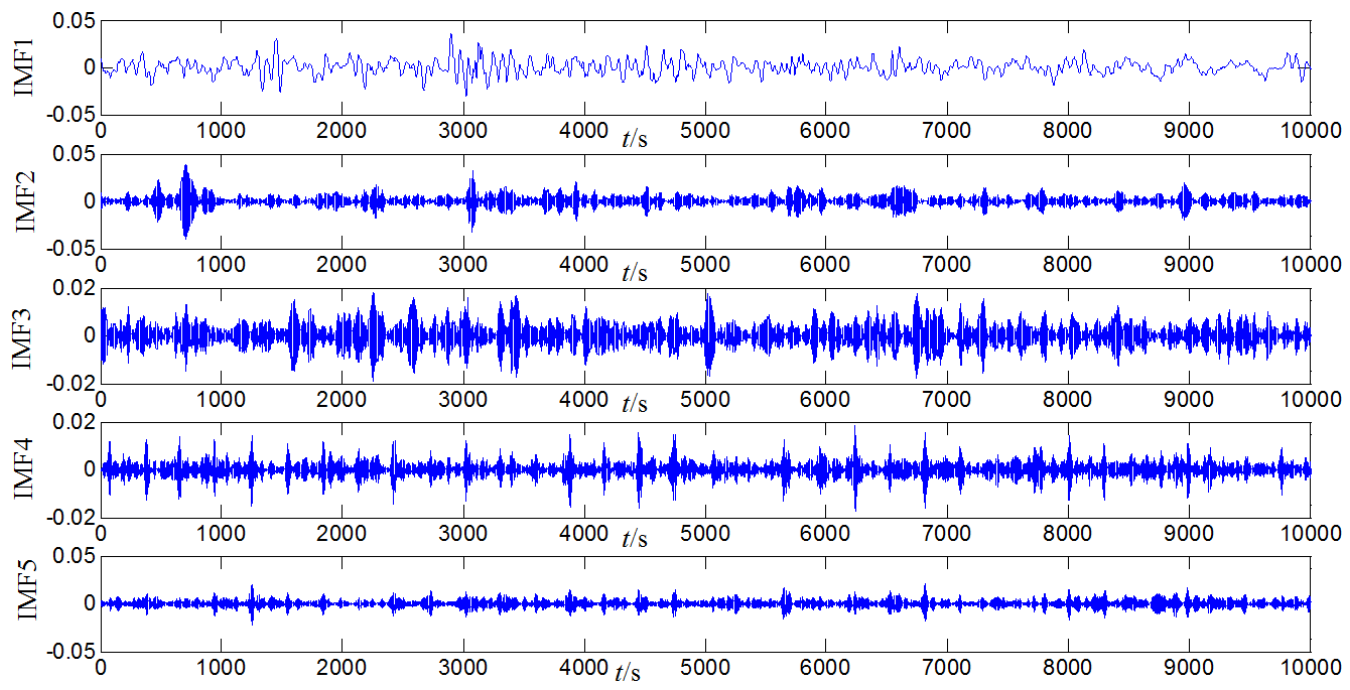

Figure 11. The decomposition result using VMD

Figure 12 (c) shows the result of the combination of IITD L-kurtosis and RDT. By comparing Figure 10 (a) and Figure 12 (a), the periodic impact characteristic is more pronounced in the latter graphic. Meanwhile, by comparing Figure 12 (b) and Figure 12 (c), it can be seen that the faulty feature frequency $(87.04 \mathrm{~Hz})$ and its 2-5 harmonics $(174.1 \mathrm{~Hz}, 261.1 \mathrm{~Hz}$, $351.9 \mathrm{~Hz}, 437 \mathrm{~Hz})$ are clearly extracted in the former graphic, which is matched the theoretical calculation value (show in (20)) and the out race fault is successfully detected.

Table 4. The corresponding L-Kurtosis values.

\begin{tabular}{llllll}
\hline IMF & IMF1 & IMF2 & IMF3 & IMF4 & IMF5 \\
\hline L-Kurtosis value & 3.7599 & 7.8067 & 3.9540 & 5.7734 & 4.5223 \\
\hline
\end{tabular}

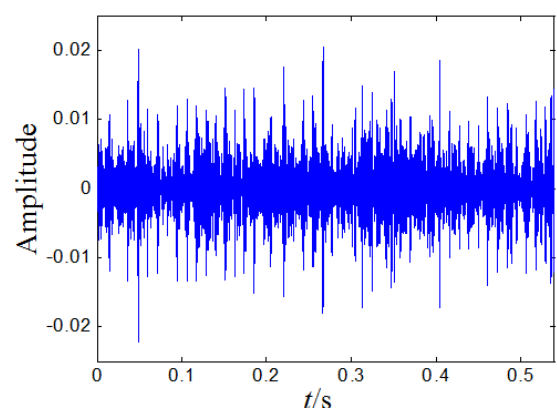

(a)

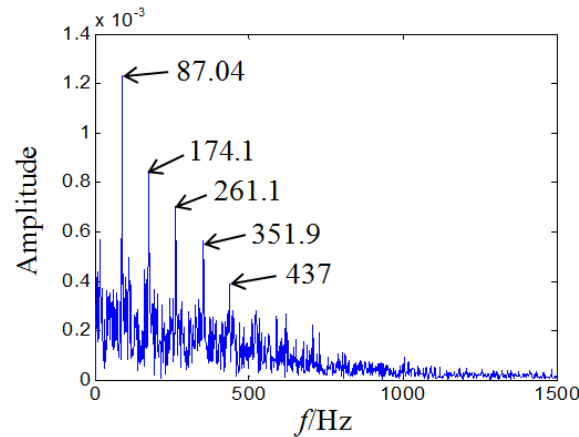

(b)

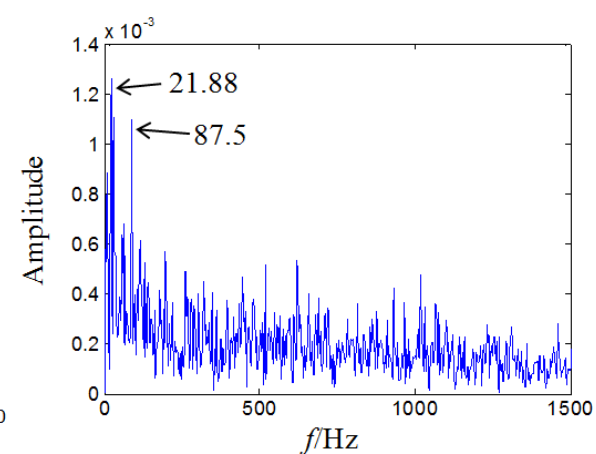

(c)

Figure 12. The time domain waveform of the optimal IMF and corresponding Hilbert envelope spectrum, (c) the combination of IITD, L-kurtosis and RDT. 


\subsection{Gear Broken Teeth Fault Detection}

The time domain waveform of the raw vibration signal and corresponding Hilbert envelope spectrum are presented in Figure 13. From Figure 13 (b), the shafting frequency $(29.63 \mathrm{~Hz})$ and its $2-3$ harmonics $(59.26 \mathrm{~Hz}, 88.89 \mathrm{~Hz})$ are shown clearly, but the other harmonics are submerged.

The next procedures are the same as the part 4.1 and the relative L-Kurtosis values of decomposed IMFs are shown in Table 5. For the optimal IMF, its time domain waveform is shown in Figure 14 (a) and the periodic impact characteristic is clearer. The Hilbert envelope spectrum of the optimal IMF is shown in Figure 14 (b). As shown in Figure 14 (b), the shaft

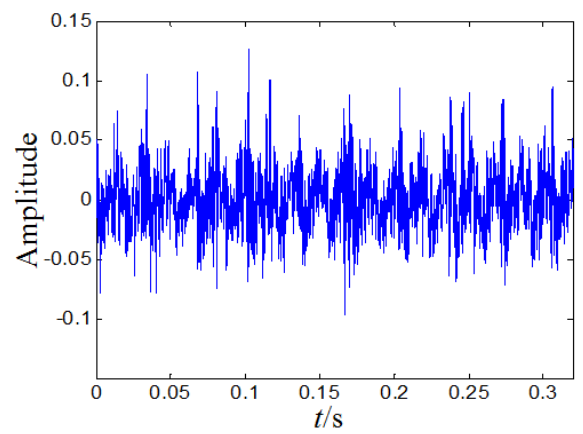

(a) rotating frequency $(29.63 \mathrm{~Hz})$ and its 2 to 9 harmonics $(59.26 \mathrm{~Hz}, 88.89 \mathrm{~Hz}, 118.5 \mathrm{~Hz}, 146.3 \mathrm{~Hz}, 175.9 \mathrm{~Hz}, 205.6 \mathrm{~Hz}$, $235.2 \mathrm{~Hz}, 264.8 \mathrm{~Hz}$ ) are all extracted clearly compared with Figure 13 (b), which are matched the shaft rotating frequency. From Figure 13 (b) and Figure 14 (b), it can be seen that the gear mesh frequency (show in (21)) are not shown.

In order to further indicate the performance of the proposed approach, Figure 14 (c) shows the result of the combination of IITD, L-kurtosis and RDT, and the faulty feature frequency can not been extracted effectively. Based on the above analysis, the teeth fault is successfully detected and the necessity of the combination of three techniques is further indicated.

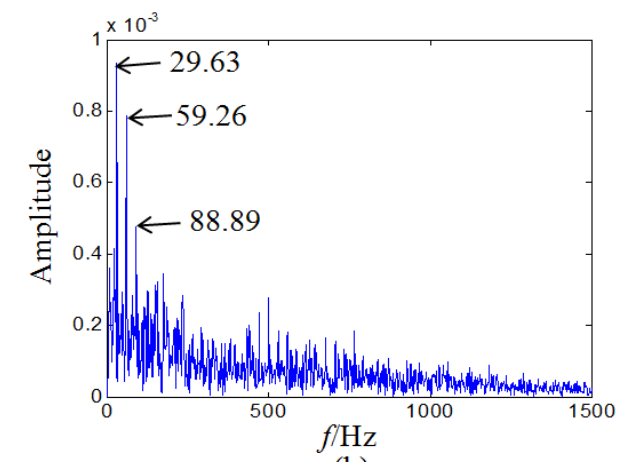

(b)

Figure 13. The time domain waveform of the raw vibration signal and corresponding Hilbert envelope spectrum.

Table 5. The corresponding L-Kurtosis values.

\begin{tabular}{llllll}
\hline IMF & IMF1 & IMF2 & IMF3 & IMF4 & IMF5 \\
\hline L-Kurtosis value & 2.6050 & 5.9214 & 5.6149 & 6.5065 & 11.2665 \\
\hline
\end{tabular}

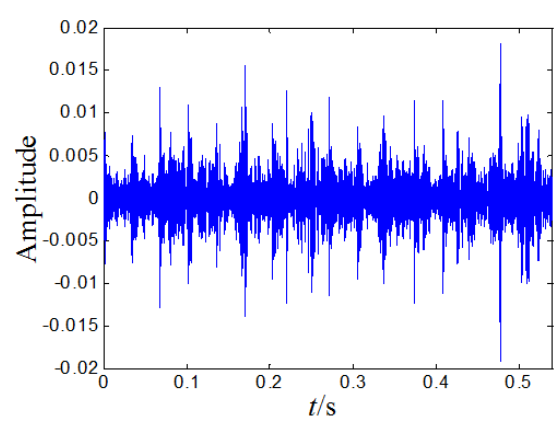

(a)

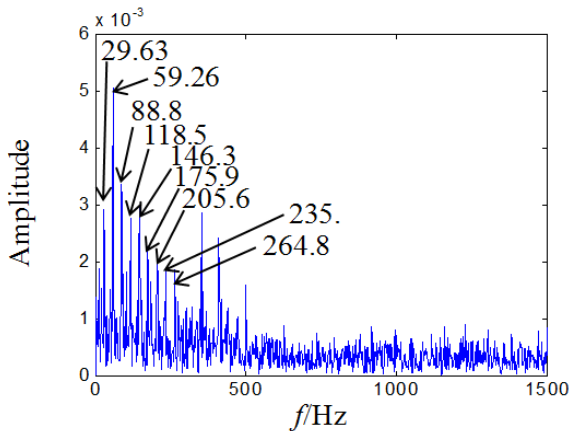

(b)

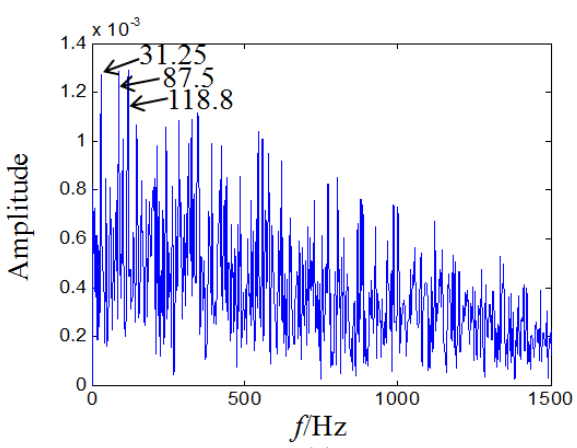

(c)

Figure 14. The time domain waveform of the optimal IMF and corresponding Hilbert envelope spectrum, (c) the combination of IITD, L-kurtosis and RDT.

\section{Conclusion}

In this paper, a novel hybrid approach is proposed for detecting the faults for rotating machinery. The non-stationary signal decomposition capability of VMD is explored in fault detection. However, aiming at the rotating machinery like rolling element bearing and gear, the faulty information is commonly located at the high frequency band, it is difficult to determine which decomposed IMF the faulty information exists in. The introduction of L-Kurtosis overcomes the problem that the selection of the optimal IMF depends on human experience. Meanwhile, L-Kurtosis combines the signal decomposition advantages of VMD and signal purification ability of RDT, and the faulty feature frequency is extracted by Hilbert envelope analysis.

Through analyzing the simulated data and real experimental data collected from the bearings and gear with different types of faults, the accuracy of the proposed approach is demonstrated. Therefore, this approach has promising applications in condition detecting of mechanical system.

\section{Acknowledgements}

This work was supported by the National Natural Science 
Foundation of China (Grant No. 11172131) and the Priority Academic Program Development of Jiangsu Higher Education Institute.

\section{References}

[1] Y. Zhang, and R. Randall, "Rolling element bearing fault diagnosis based on the combination of genetic algorithms and fast kurtogram," Mech. Syst. Signal Pr., vol. 23, no. 5, pp. 1509-1517, July 2009.

[2] M. Riera-Guasp, J. A. Antonino-Daviu, G. A. Capolino, "Advances in electrical machine, power electronic, and drive condition monitoring and fault detection: state of the art," IEEE Trans. Ind. Electron., vol. 62, no. 3, pp. 1746-1759, March 2015 .

[3] Y. X. Wang, J. W. Xiang, R. Markert, and M. Liang, "Spectral kurtosis for fault detection, diagnosis and prognostics of rotating machines: a review with applications," Mech. Syst. Signal Process., vol. 66-67, pp. 679-698, January 2016.

[4] J. Liu, and Y. M. Shao, "Dynamic modeling for rigid rotor bearing systems with a localized defect considering additional deformations at the sharp edges," J. Sound Vib., vol. 398, pp. 84-102, June 2017.

[5] M. Cerrada, R. V. Sanchez, and C. Li, et al., "A review on data-driven fault severity assessment in rolling bearings," Mech. Syst. Signal Process., vol. 99, pp. 169-196, January 2018.

[6] J. Xiang, Y. Zhong, and H. Gao, "Rolling element bearing fault detection using PPCA and spectral kurtosis," Measurement., vol. 75, pp. 180-191, November 2015.

[7] S. L. Lu, P. Zheng, and Y. B. Liu, "Sound-aided vibration weak signal enhancement for bearing fault detection by using adaptive stochastic resonance," j. Sound Vib., vol. 449, pp. 18-29, Juny 2019.

[8] Z. W. Liu, Z. J. He, W. Guo, and Z. C. Tang, “A hybrid fault diagnosis method based on second generation wavelet de-noising and local mean decomposition for rotating machinery," ISA T., vol. 61, pp. 211-220, March 2016.

[9] H. Liu, and J. W. Xiang, "Kernel regression residual decomposition-based synchroextracting transform to detect faults in mechanical systems," ISA T., vol. 87, pp. 251-263, April 2019.

[10] H. M. Zhao, H. L. Liu, and J. J. et al., "Research on a fault diagnosis method of rolling bearings using variation mode decomposition and deep belief network," J. Mech. Sci. Technol., vol. 33, no. 9, pp. 4165-4172, September 2019.

[11] K. X. Hong, and G. X. Lin, "State classification of transformers using nonlinear dynamic analysis and Hidden Markov models," Measurement, vol. 147, Dec 2019.

[12] J. Y. Jiao, M. Zhao, J. Lin, Ding, and C. C. Ding, "Deep coupled dense convolutional network with complementary data for intelligent fault diagnosis," IEEE T. Ind. Electron., vol. 66, no. 12, pp. 9858-9867, December 2019.

[13] J. W. Xiang, and Y. T. Zhong, "A fault detection strategy using the enhancement ensemble empirical mode decomposition and random decrement technique," Microelectronics Reliab., vol. 75, pp. 317-326, August 2017.
[14] N. E. Huang, Z. Shen, and S. R. Long, et al., "The empirical mode decomposition and the Hilbert spectrum for nonlinear and non-stationary time series analysis," Proc. Royal Soc. A: Math., Phys. Eng. Sci., vol. 454, no. 1971, pp. 903-995, March 1998.

[15] K. Dragomiretskiy, and D. Zosso, "Variational mode decomposition,” IEEE Trans. Signal Process., vol. 62, no. 3, pp. 531-544, February 2014.

[16] X. Zheng, Q. Zhou, N. Zhou, R. J. Liu, Z. Y. Hao, and Y. Qiu, "A dichotomy-based variational mode decomposition method for rotating machinery fault diagnosis," Meas. Sci. Technol., vol. 31, no. 1, pp. 015003, January 2019.

[17] R. Gu, J. Chen, R. J. Hong, H. Wang, and W. W. Wu, "Incipient fault diagnosis of rolling bearings based on adaptive variational mode decomposition and Teager energy operator," Measurement, vol. 149, January 2019.

[18] X. W. Du, G. R. Wen, and D. Liu, et al., "Fractional iterative variational mode decomposition and its application in fault diagnosis of rotating machinery," Meas. Sci. Technol., vol. 30, no. 12, December 2019.

[19] C. Yang, and M. P. Jia, "A novel weak fault signal detection approach for a rolling bearing using variational mode decomposition and phase space parallel factor analysis," Meas. Sci. Technol., vol. 30, no. 11, November 2019.

[20] C. S. Withers, and S. Nadarajah, "Bias-reduced estimates for skewness, kurtosis, L-skewness and L-kurtosis," J. Stat. Plan. Infer., vol. 141, no. 12, pp. 3839-3861, December 2011.

[21] S. P. Liu, S. M. Hou, K. D. He, and W. H. Yang, "L-Kurtosis and its application for fault detection of rolling element bearings," Measurement, vol. 116, pp. 523-532, February 2018.

[22] H. A. Cole, "On-the-line analysis of random vibrations," AIAA Pap., vol. 68, pp. 288-319, 1971.

[23] M. J. Desforges, J. E. Cooper, and J. R. Wright, "Spectral and modal parameter estimation from output-only measurements," Mech. Syst. Signal Pr., vol. 9 no. 2, pp. 169-186, March 1995.

[24] S. R. Ibrahim, K. R. Wentx, and J. Lee, "Damping identification from non-linear random response using a multi-triggering random decrement technique," Mech. Syst. Signal Pr., vol. 1, no. 4, pp. 389-397, October 1987.

[25] S. V. Modak, C. Rawal, and T. K. Kundra, "Harmonics elimination algorithm for operational modal analysis using random decrement technique," Mech. Syst. Signal Pr., vol. 24, no. 4, pp. 922-944, May 2010.

[26] J. C. Asmussen, R. Brincker, and S. R. Ibrahim, "Statistical theory of the vector random decrement technique," J. Sound Vib., vol. 226, no. 2, pp. 329-344, September 1999.

[27] J. K. Vandiver, A. B. Dunwoody, and R. B. Campbell, “A mathematical basis for the random decrement vibration signature analysis technique," J. Mech. Design., vol. 104, pp. 307-313, 1982 .

[28] Z. W. Huang, Y. Z. Li, X. G. Hua, Z. Q. Chen, and Q. Wen, "Automatic Identification of Bridge Vortex-Induced Vibration Using Random Decrement Method," Appl. Sci-basel., vol. 9, no. 10, May 2019.

[29] S. R. Ibrahim, "The use of random decrement technique for identification of structural modes of vibration,", AIAA Pap., vol. 77, pp. 1-9, 1977. 
[30] S. R. Ibrahim, "Random decrement technique for modal identification of structures," J. Spacecr. Rocket., vol. 14, pp. 696-700, 1977.

[31] A. N. Tichonov, "Solution of incorrectly formulated problems and the regularization method," Soviet Math., vol. 4, pp. $1035-1038,1963$
[32] D. P. Bertsekas, "Multiplier methods: A survey," Automatica., vol. 12, no. 2, pp. 133-145, 1976.

[33] L. L. Cui, and Y. Zhang, et al., "Application of pattern recognition in gear faults based on the matching pursuit of a characteristic waveform," Measurement, vol. 104, pp. 212-222, July 2017. 